\title{
Philosophiques
}

Patrice Vermeren, Le rêve démocratique de la philosophie

d'une rive à l'autre de l'Atlantique, suivi de Essai de philosophie populaire, par Amédée Jacques. Postface de Arturo Andres Roig, Paris, L'Harmattan, collection « La philosophie en commun ", 325 pages.

\section{Laurent Fedi}

Volume 29, numéro 2, automne 2002

La démocratie délibérative

URI : https://id.erudit.org/iderudit/006285ar

DOI : https://doi.org/10.7202/006285ar

Aller au sommaire du numéro

Éditeur(s)

Société de philosophie du Québec

ISSN

0316-2923 (imprimé)

1492-1391 (numérique)

Découvrir la revue

Citer ce compte rendu

Fedi, L. (2002). Compte rendu de [Patrice Vermeren, Le rêve démocratique de la

philosophie d'une rive à l'autre de l'Atlantique, suivi de Essai de philosophie

populaire, par Amédée Jacques. Postface de Arturo Andres Roig, Paris,

L'Harmattan, collection " La philosophie en commun », 325 pages.]

Philosophiques, 29(2), 395-396. https://doi.org/10.7202/006285ar d'utilisation que vous pouvez consulter en ligne. 
Patrice Vermeren, Le rêve démocratique de la philosophie d'une rive à l'autre del'Atlantique, suivi de Essai de philosophie populaire, par Amédée Jacques. Postface de Arturo Andres Roig, Paris, L'Harmattan, collection « La philosophie en commun », 325 pages.

Ce livre est une enquête - passionnante et magistralement menée - sur A médée Jacques, professeur de philosophie du XIX ${ }^{e}$ siècle destitué de ses fonctions en 1850 sous un gouvernement conservateur, pour le crime d'avoir dirigé une revue républicaine. Ce récit, ouvert sur une problématique philosophique, s'inscrit dans le cadre de la réflexion menée depuis plus de vingt ans par Patrice Vermeren au sujet des paradoxes de l'institution philosophique.

A médée J acques a été formé à la philosophie dans les structures d'enseignement édifiées par Victor Cousin. A près avoir enseigné dans les collèges royaux d'A miens et de D ouai, ainsi qu'aux collèges Bourbon et $\mathrm{H}$ enri IV comme suppléant, et à $\mathrm{N}$ ormale comme chargé de cours, il obtient un poste de titulaire à Versailles et enfin au collège Louis-Le-Grand de Paris. II a publié en 1846 un M anuel de philosophie à l'usage des collèges approuvé, sous la monarchie bourgeoise de Louis-Philippe, par le Conseil royal de l'instruction publique. II a collaboré au D ictionnaire des sciences philosophiques d'A dol phe Franck, l'encyclopédie de l'école éclectique. II est égal ement I'auteur de plusieurs mémoires académiques, dans lesquels on chercherait en vain les ferments de la subversion. Ce «professeur distingué, si parfaitement universitaire» (p. 32), désireux de sortir des ornières du cousinisme, fonde en 1847 sa revue, La Liberté de Penser. Avec la révolution de février 1848 s'ouvre une ère nouvelle, du moins le croit-on. Il va de soi pour A médée Jacques que la philosophie, dont le statut est lié à la prise de parole publique, doit entrer sur scène, fût-ce sur le mode du discours théorique. II brandit alors la bannière de la défense de la philosophie, et désigne deux adversaires : le parti clérical, anti-républicain en son principe, et les utopies égalitaristes. Pour lui, la philosophie enseignée ne doit pas être une doctrine parmi d'autres, encore moins une doctrine d'Etat, mais l'ensemble des vérités fondamentales, qui sont aussi (et c'est là le nerf de ses convictions) les vérités fondatrices de la vie démocratique. En mars 1848 est fondée la Société démocratique des libres-penseurs. A médée J acques en sera le vice-président, et son ami Jules Simon, le président. $M$ ais après le retour à l'ordre et la loi Falloux de 1850, la réaction ne se fait pas attendre. Le professeur J acques subit même sort que J oseph Ferrari. Puis le coup d'Etat du 2 décembre entérine le naufrage des libertés publiques et sonne le glas des revues républicaines.

À partir de cette «affaire», P. Vermeren s'interroge sur le statut du professeur de philosophie dans l'Etat libéral naissant et sur la mission de la philosophie eu égard aux droits de la «raison ». M ais surtout il met en question la viabilité de l'Etat idéal et philosophique dont rêve A médée J acques : «La vérité politique de la philosophie devenue une affaire de l'Etat ne serait-elle pas finalement celle-ci : que l'Etat moderne est effectivement impossible?» (p. 48).

C'est alors que la seconde partie du parcours que nous propose Patrice Vermeren, plus exotique, prend tout son sens. Amédée Jacques, expatrié en A mérique latine, va tenter de réaliser le « rêve démocratique de la philosophie» dans l'A rgentine de Bartolomé $M$ itre. L'aventure latino-américaine débute par la tentative de créer en Uruguay un cours de sciences. A médée Jacques, pourtant formé dans l'école «spiritualiste» de Victor Cousin, se passionne pour les sciences positives, pour lesquelles il a des aptitudes que ses élèves lui reconnaîtront sans conteste; à tel point que certains 


\section{$396 \cdot$ Philosophiques / Automne 2002}

commentateurs d'O utre-A tlantique verront en lui un vrai «positiviste». Ce n'est pas là le moindre de ses paradoxes. Cet homme du XIX e siècle, progressiste, ne pouvait croire en la «modernité» sans adhérer à l'expansion des sciences et des techniques. $M$ ais ici, on pressent quelque chose de plus, qui relève de l'articulation des sciences à la philosophie et à la politique de l'émancipation. Parmi les textes placés en annexe, les longues descriptions des bords du Salado, mêlant géographie, zoologie, histoire et économie sur le mode tantôt scientifique, tantôt pittoresque, ne sont-elles pas significatives d'une pratique de pensée bouleversant les schémas de la philosophie abstraite? La veine épistémologique d'A médée J acques apparaît de temps à autre dans l'exposé de $P$. Vermeren, et l'on y sent un motif important, qui eût mérité sans doute un examen plus systématique. Q uoi qu'il en soit, il est clair que Jacques n'était pas ce baroudeur alcoolique dépeint par la plume revancharde des Goncourt.

Si P. Vermeren réhabilite manifestement le défenseur de la liberté victime des pouvoirs, ce n'est jamais au détriment des contextes. Ce livre est, en son inspiration philosophique, un essai critique sur les ambiguïtés de la philosophie institutionnalisée. Et l'auteur prend ses distances vis-à-vis de son objet pour montrer en quoi le rêve d'un Etat libéral et philosophe est irréaliste. D'autre part, l'idéal émancipateur de Jacques va de pair avec une certaine idée de la civilisation bien ancrée dans le XIX ${ }^{\mathrm{e}}$ siècle, et rencontre en ce point ses propres limites. "A médée Jacques avait lutté en France au nom de la République pour la souveraineté du peuple. Son adhésion à l'idée d'un Etat démocratique libéral naissant en Argentine lui fait rejeter la différence sauvage; et pour maintenir son idéal égalitaire, il lui faut exclure du peuple l'Indien en tant qu'animal féroce, par la négation de son humanité» (p. 83).

Toujours est-il qu'A médée Jacques réalise son objectif en prenant la charge du Collège national de Buenos Aires. Dans l'expérience concrète de ce professeur hors pair, P. Vermeren perçoit la mise en application d'une philosophie de l'éducation : refus de la spécialisation précoce, respect du développement naturel de l'intelligence, nécessité d'une culture encyclopédique et d'un décloisonnement des savoirs. M ais J acques a-t-il été l'instituteur du peuple, ou a-t-il exporté un modèle français d'éducation?

Les précieux textes d'A médée Jacques placés en annexe occupent plus de la moitié de l'ouvrage. O n retiendra, parmi ces documents rares, une très intéressante discussion tripartite entre le professeur berlinois Karl M ichelet, Victor Cousin et A médée Jacques au sujet du «panthéisme», ou encore une lettre d'A médée J acques à Sarmiento qui témoigne de certaines convergences de vues.

Insistons, enfin, sur une des qualités majeures de ce livre : il réussit à communiquer au lecteur le plaisir du chercheur sillonnant le R io de la Plata sur les traces du philosophe émigré et «rêveur».

LAURENT FEDI.

CNRS - Université de Lille 2

\section{Jean-Claude Guillebaud, Le principe d'humanité, Paris, Seuil, 2001, 380 p.}

Jean-Claude G uillebaud est une figure familière dans le monde intellectuel de langue française dont le parcours impressionnant mérite d'être brièvement rappelé. A ncien grand reporter, devenu éditorialiste pour le quotidien Sud O uest, il fut également journaliste pour L e M onde et le $\mathrm{N}$ ouvel $\mathrm{O}$ bservateur ainsi que président des Reporters sans frontières. De nombreuses distinctions ont ponctué sa carrière journalistique 